\title{
Sample preparation artifacts in nuclear materials and mitigation strategies
}

\author{
A. Aitkaliyeva ${ }^{1}$, J. W. Madden ${ }^{1}$, B. D. Miller ${ }^{1}$, J. I. Cole ${ }^{1}$, J. Gan $^{1}$ \\ ${ }^{1}$ Idaho National Laboratory, Idaho Falls, Idaho, 83415
}

Diverse microstructures form in nuclear materials upon exposure to radiation. The defects produced during irradiation of materials can alter their mechanical properties and lead to embrittlement of reactor structural materials during service life. Therefore, it is imperative to know various radiation effects in reactor materials since it can aid in understanding in-reactor degradation behavior, accounting for irradiation effects in design, and producing new generation radiation-tolerant materials. Characterization of radiation-induced changes in reactor materials at the nano and atomic scales is typically conducted in transmission electron microscopes (TEM). Three most commonly used sample preparation techniques include electro-polishing, broadbeam ion milling, and focused ion beam (FIB) approach. However, preparation of samples using conventional sample preparation techniques, such as electro-polishing and ion milling, requires close-in, hands-on manipulation of the sample for extended periods of time. This is not feasible with highly radioactive nuclear materials.

Electro-polishing involves the usage of electrolytic solution and doesn't introduce any defects that can be confused with irradiation-induced defects, and therefore it has traditionally been preferred sample preparation method for irradiated materials. Over the past several years, the popularity of electro-polishing began to diminish and FIB-based lift-out technique became preferable preparation techniques for irradiated materials. Figure 1 compares microstructure of unirradiated MA957 specimen prepared for TEM analysis using a) electro-polishing and b) FIBbased lift-out technique. Both techniques yield good quality specimens without any notable sample preparation artifacts in case of MA957.

FIB has a major disadvantage when it comes to nuclear materials characterization - beam damage. Material removal in FIB is achieved via Ga ion milling, which can lead to formation of various defects in the target material such as vacancies, interstitials, defect clusters, intermetallic phases, and Ga precipitates. The extent of sample preparation-induced damage can interfere with specimen analysis and in case of nuclear materials preclude accurate defect microstructure characterization.

Damage accumulated during FIB-based sample preparation can look very similar to the damage produced in nuclear materials irradiated to low displacements per atom (dpa) levels and/or lower temperatures. Minimization of FIB damage is typically achieved by using low-energy ( $5 \mathrm{keV}$ and $2 \mathrm{keV}$ ) cleaning. However, in some materials even extensive $2 \mathrm{keV}$ cleaning step is not sufficient in elimination of Ga damage. A number of unirradiated materials were examined and the results 
indicate that FIB damage is more extensive in some materials than the others. These formed damaged layers, consisting of both amorphous material and implanted $\mathrm{Ga}$ ions, should be eliminated in order to perform an in-depth examination of material microstructure after irradiation. The obtained results show that the energy of impinging particles should be reduced to below $2 \mathrm{keV}$ for complete elimination of sample preparation artifacts. However, only the most advanced FIB systems are capable of reducing ion energy below $2 \mathrm{keV}$ and these systems are not optimal for preparation of highly activated materials due to shorter working distances of $2 \mathrm{~mm}$ (as opposed to $10 \mathrm{~mm}$ in FEI Quanta microscopes). Therefore, post-processing of the FIB lamella has to be considered to minimize or eliminate this damage and ensure high quality analysis results.

Post-FIB processing can be achieved using a variety of advanced ion milling systems, such as PIPS-2 and NanoMill, in which energy of the Ar ion beam can be reduced to $<500 \mathrm{eV}$. Figure 2 illustrates advantages of adding post-processing to the FIB preparation procedure by providing comparison of $\mathrm{Fe}-12 \mathrm{Cr}$ specimen microstructure (a) after jet-polishing, (b) FIB-based lift-out, and (c) a combination of FIB-based lift-out technique with post-processing in PIPS-2.

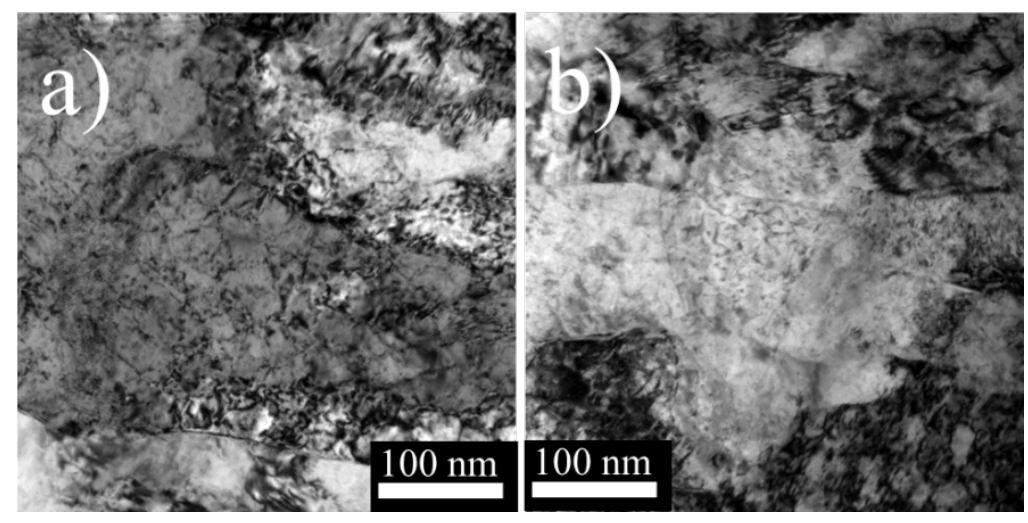

Figure 1. Bright-field TEM micrographs of unirradiated MA957 after: a) electro-polishing and b) preparation in FIB tool.
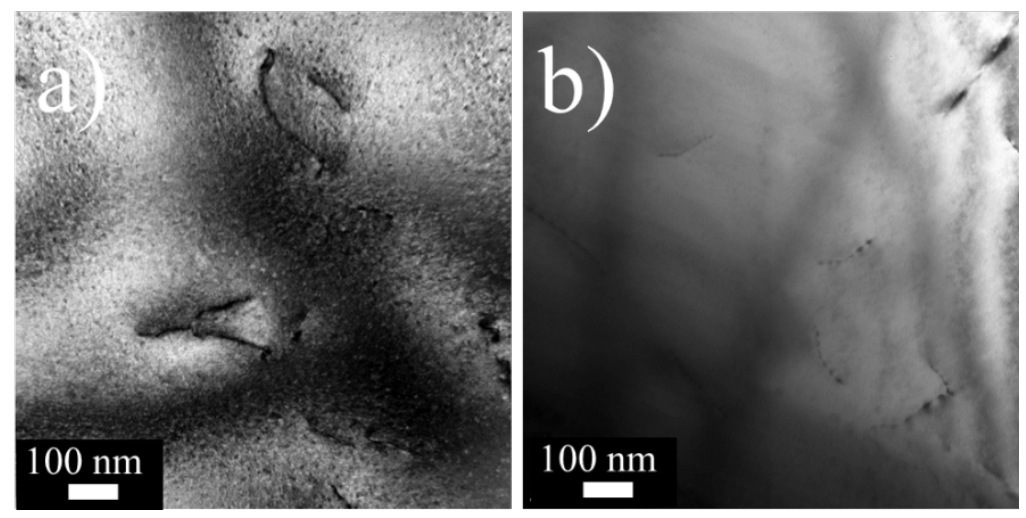

Figure 2. Bright field TEM micrographs of unirradiated Fe-12Cr sample prepared using: a) FIB and b) FIB with post-processing in PIPS-2. 